\title{
Mathematical modeling of temperature fields and concrete strength during winter concreting of building structures
}

\author{
Alexey Mestnikov ${ }^{1}$, Gavril Turantaev ${ }^{1}$, and Valeriy Fedorov ${ }^{1, *}$ \\ ${ }^{1}$ North-Eastern Federal University named after M.K. Ammosov, Institute of Engineering \& \\ Technology, Belinskogo St. 58, Yakutsk, 677027, Russia
}

\begin{abstract}
In this paper we present a mathematical model of a threedimensional non-stationary temperature field for concrete that hardens in winter conditions. The developed mathematical model describes heat transfer processes basing on the differential heat equation. We also consider the classical boundary conditions of the I, II, III and IV kinds. We compiled a computer interpolation of the family of nomograms in the second order regression equation in order to calculate the relative theoretical strength of concrete after heat treatment.
\end{abstract}

\section{Introduction}

Given the climatic conditions of the Arctic zone of the Russian Federation and the world, the concrete works are often carried out in the winter [1]. For example, in Siberia and the Far North about $50-75 \%$ of the annual volume of such works is performed in the winter construction period [2,3].

As is it known, when concreting any building structures, regardless the accepted method of work, temperature stresses occur in concrete, associated with uneven temperature field along the cross section of the structure [4-6]. An important task is to provide the thermal stress state of the structure, in which no destructive processes occur in concrete. The recommendations of the Russian and foreign researchers [7-16] on this issue are insufficiently justified and present restrictions on temperature variations within the cross section of the structure, which are difficult to implement practically. To prevent unacceptable thermal stresses in concrete during its heat treatment one must avoid its overheating or underheating. Also one must avoid limiting rates of the concrete temperature growth after the beginning of its heat treatment and limiting rates of the concrete temperature decrease after the end of its heat treatment for fragments of the concreted structures [17-22]. Consequently, development of reliable and accurate methods for calculating the temperature fields in concrete, as well as assessment of its theoretical relative strength after the heat treatment process are of immediate interest even at the present time.

\footnotetext{
* Corresponding author: valeriyif.ykt@gmail.com
} 


\section{Methods}

While performing the mathematical modeling of temperature fields we used the classical equations of thermal physics which do not require proof of reliability [2, 23]:

1) Differential equation of heat conduction for previously laid concrete, to which the concreted fragments are adjacent.

2) The same with a space-distributed heat source of exothermic kind for fresh concrete.

3) Classical boundary conditions of the kinds I (given the temperature of the concrete at the boundary), II (given the intensity of heat flow at the boundary), III (heat transfer at the boundary according to Newton's law), and IV (condition of thermal contiguity of two solids).

Differential equations are obtained by the fractional step method [24]. The calculation of the theoretical relative strength of concrete after heat treatment was performed according to the procedure described in TSN 12-336-2007 [25]. The computer interpolation of experimental nomograms was performed using the mathematical package MathCAD 2001i Professional. To automate the calculation of the theoretical strength of concrete, an easy-touse calculator has been developed using the Microsoft Office EXCEL 2007 software, taking into account the regulations of TSN 12-336-2007.

\section{Results and Discussion}

In accordance with Fig. 1, fragments of concreted elements are spatial structures, which predetermines the necessity for setting a three-dimensional problem. In this formulation, a fragment of a wall with a thickness $\triangle$ of length $\mathrm{L}$ and height $B$ is concreted at a negative equivalent temperature $\theta_{v}(\tau)$. On the left, on the right and on the bottom, this fragment adjoins the previously erected sections of the wall, sustained for a long time and reached a temperature $\theta_{e}(\tau)$. If we accept the origin of coordinates, as shown in Fig. 1, the thermal influence of the concreted fragment on the previously erected construction sections is characterized by the depth of thermal influence along the $O Y$ and $O Z$ axes ( $Y Y$ and $Z Z$, respectively).

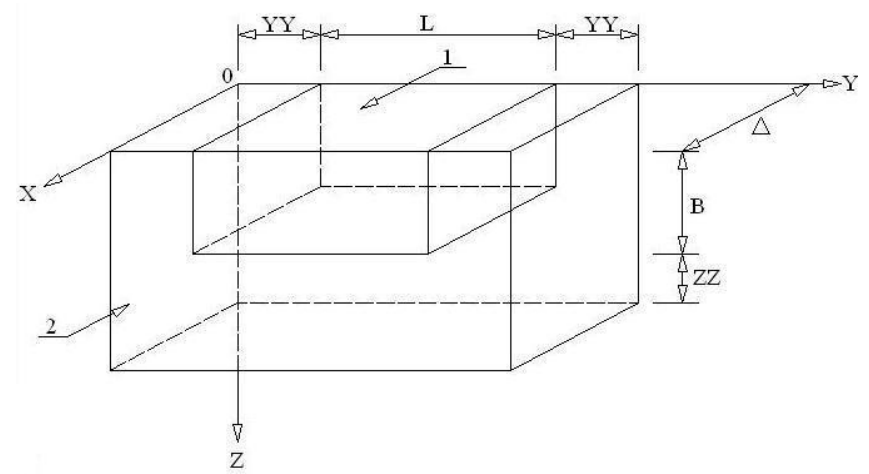

Fig. 1. The calculation scheme of the concreted fragment of the wall (1) adjacent to the previously erected sections of the wall (2): YY, ZZ are zones of thermal influence on the previously erected fragments of walls along the axes $\mathrm{Y}$ and $\mathrm{Z}$

Initially $(\tau=0)$ we can take $Y Y=\frac{L}{2}$ and $Z Z=\frac{B}{2}$, but further we calculations should be performed to specify the actual existing depth of the heat influence.

The reduced heat transfer coefficient $K_{\text {red }}\left(\mathrm{W} /\left(\mathrm{m}^{2} \cdot \mathrm{K}\right)\right)$ is equal to:

1. For side faces $\left(\mathrm{K}_{\text {red1 }}\right)$ of the area $F_{1}$ each $\left(\frac{1}{\alpha_{\text {wind }}}+\sum_{i}\left(\frac{\delta_{i}}{\lambda_{i}}\right)\right)^{-1}$. 
2. For upper face $\left(\mathrm{K}_{\mathrm{red} 2}\right)$ of the fragment $F_{2}\left(\frac{1}{\alpha_{\text {wind }}}+\frac{\delta_{y}}{\lambda_{y}}\right)^{-1}$.

Where $\sum_{i}\left(\frac{\delta_{i}}{\lambda_{i}}\right)$ is total thermal resistance of the formwork $\left(\mathrm{m}^{2} \cdot \mathrm{K} / \mathrm{W}\right)$;

$\frac{\delta_{y}}{\lambda_{y}}$ is the insulation thermal resistance on the upper surface $\left(\mathrm{m}^{2} \cdot \mathrm{K} / \mathrm{W}\right)$;

$\frac{1}{\alpha_{\text {wind }}}$ is the thermal resistance of the boundary layer while the wind flowing round the construction $\left(\mathrm{m}^{2} \cdot \mathrm{K} / \mathrm{W}\right)$.

Mathematical model of the considered problem can be written in the form $[2,19,20]$ :

$$
\left\{\begin{array}{l}
\frac{\partial t_{f}(x, y, z, \tau)}{\partial \tau}=\frac{\lambda_{f}}{C_{\mathrm{f}}}\left[\frac{\partial^{2} t(x, y, z, \tau)}{\partial x^{2}}+\frac{\partial^{2} t(x, y, z, \tau)}{\partial y^{2}}+\frac{\partial^{2} t(x, y, z, \tau)}{\partial z^{2}}\right]+\frac{\omega(\tau)}{C_{\mathrm{f}}} \\
x \in \triangle \wedge(Y Y \leq y \leq(Y Y+L)) \wedge z \in B
\end{array}\right.
$$

(1) - Differential heat transfer equation with a spatial-distributed heat source for a concreted fragment of structure.

$$
\left\{\begin{array}{l}
\frac{\partial t_{b}(x, y, z, \tau)}{\partial \tau}=\frac{\lambda_{b}}{C_{\mathrm{b}}}\left[\frac{\partial^{2} t(x, y, z, \tau)}{\partial x^{2}}+\frac{\partial^{2} t(x, y, z, \tau)}{\partial y^{2}}+\frac{\partial^{2} t(x, y, z, \tau)}{\partial z^{2}}\right] \\
{[x \in \triangle \wedge y \in Y Y \wedge z \in(B+Z Z)] \wedge[x \in \triangle \wedge(Y Y \leq y \leq(Y Y+L))] \wedge} \\
\wedge[B \leq z \leq(B+Z Z)] \wedge[x \in \triangle \wedge((Y Y+L) \leq y \leq(2 Y Y+L)) \wedge z \in(B+Z Z)]
\end{array}\right.
$$

(2) - Differential heat transfer equation for the previously erected fragments of structure.

$$
\begin{aligned}
& \left\{\begin{array}{l}
\frac{\partial t_{y, n}(x, y, z, \tau)}{\partial \tau}=\frac{\lambda_{y, n}}{C_{y, n}}\left[\frac{\partial^{2} t_{y, n}(x, y, z, \tau)}{\partial x^{2}}+\frac{\partial^{2} t_{y, n}(x, y, z, \tau)}{\partial y^{2}}+\frac{\partial^{2} t_{y, n}(x, y, z, \tau)}{\partial z^{2}}\right]+ \\
+\frac{q_{1}}{\Delta y \cdot \Delta z \cdot C_{y, n}}, \\
\left((x=0 \vee x=\triangle) \wedge\left(x=X_{f}, f=1, \ldots, u\right)\right) \wedge(Y Y \leq y \leq(Y Y+L)) \wedge z \in B
\end{array}\right. \\
& \left\{\begin{array}{l}
-\lambda_{f} \frac{\partial t_{f}(x, y, z, \tau)}{\partial x}+\lambda_{n} \frac{\partial t_{n}(x, y, z, \tau)}{\partial x}=0, \\
(x=0 \vee x=\triangle) \wedge(Y Y \leq y \leq(Y Y+L)) \wedge z \in B
\end{array}\right. \\
& \left\{\begin{array}{l}
-\lambda_{n} \frac{\partial t_{n}(x, y, z, \tau)}{\partial x}+\lambda_{y} \frac{\partial t_{y}(x, y, z, \tau)}{\partial x}=0, \\
(x=0 \vee x=\triangle) \wedge(Y Y \leq y \leq(Y Y+L)) \wedge z \in B
\end{array}\right.
\end{aligned}
$$

(3.1), (3.2), (3.3) are the equations of thermal adjoining of the formwork to the body of the concreted fragment in the case when the linear heat source $q_{1}$ cannot be reduced to a conditionally flat heat source $q_{o n}(\tau)$.

$$
\left\{\begin{array}{l} 
\pm \lambda_{f} \frac{\partial t_{f}(x, y, z, \tau)}{\partial x}=q_{o n}(\tau)+K_{r e d}\left[t_{f}(x, y, z, \tau)-\theta_{e}(\tau)\right] \\
(x=0 \vee x=\triangle) \wedge(Y Y \leq y \leq(Y Y+L)) \wedge z \in B
\end{array}\right.
$$


(3.4) is the same equation in the case when the linear heat source can be reduced to a conditionally flat heat source, is the combination of boundary conditions of the II and III kinds.

$$
\begin{aligned}
& \left\{\begin{array}{l}
-\lambda_{b} \frac{\partial t_{b}(x, y, z, \tau)}{\partial x}=\alpha_{\text {wind }}\left[t_{b}(x, y, z, \tau)-\theta_{e}(\tau)\right] \\
{[(x=0 \vee x=\triangle) \wedge y \in Y Y \wedge z \in(B=Z Z)] \wedge[(x=0 \vee x=\triangle) \wedge(Y Y \leq y \leq(Y Y+L)) \wedge} \\
\wedge(B \leq z \leq(B+Z Z))] \wedge[(x=0 \vee x=\triangle) \wedge(Y Y+L) \leq y \leq(2 Y Y+L) \wedge z \in(B+Z Z)]
\end{array}\right. \\
& \left\{\begin{array}{l}
-\lambda_{b} \frac{\partial t_{b}(x, y, z, \tau)}{\partial z}+\lambda_{f} \frac{\partial t_{f}(x, y, z, \tau)}{\partial z}=0, \\
x \in \triangle \wedge(y \in Y Y \vee(Y Y+L) \leq y \leq(2 Y Y+L)) \wedge z=B \\
x \in \triangle \wedge(y=Y Y \vee y=(Y Y+L)) \wedge z \in B
\end{array}\right.
\end{aligned}
$$

(5) are boundary conditions of the IV kind at the borders of the thermal contact of the constructed fragment and previously erected construction fragments.

$\left\{\begin{array}{l}-\lambda_{f} \frac{\partial t_{f}(x, y, z, \tau)}{\partial z}=K_{r e d 2}\left(t_{f}(x, y, z, \tau)\right)-\theta_{e}(\tau) \\ x \in \triangle \wedge(Y Y \leq y \leq(Y Y+L)) \wedge z=0\end{array}\right.$

(6) are boundary conditions of the III kind on the lateral surfaces of the concreted fragment.

$$
\begin{aligned}
& \left\{\begin{array}{l}
t_{f}(x, y, z, \tau)=\text { const }=\theta_{e}(\tau), \\
\{[x \in \triangle \wedge y \in Y Y \wedge z \in(B+Z Z)] \wedge[x \in \triangle \wedge(Y Y \leq y \leq(Y Y+L)) \wedge B \leq z \leq(B+ \\
+Z Z)] \wedge[x \in \triangle \wedge((Y Y+L) \leq y \leq(2 Y Y+L)) \wedge z \in(B+Z Z)]\}, \tau=0
\end{array}\right. \\
& \left\{\begin{array}{c}
t_{f}(x, y, z, \tau)=\text { const }=t_{\text {wind }}, \\
x \in \triangle \wedge(Y Y \leq y \leq(Y Y+L)) \wedge z \in B \wedge \tau=0
\end{array}\right.
\end{aligned}
$$

(7), (8) are initial conditions.

$$
\left\{\begin{array}{l}
t(x, y, z, \tau)=\text { const }=\theta_{e}(\tau) \\
{[x \in \triangle \wedge(y=0) \vee(y=(L=2 Y Y)) \wedge z \in(B+Z Z)] \wedge[x \in \triangle \wedge y \in(L+2 Y Y) \wedge z=(B+Z Z)]}
\end{array}\right.
$$

(9) are boundary conditions of the I kind at the end of the zones of thermal influence of the concreted fragment.

$\frac{\partial t_{f, b}(x=\Delta / 2, y, z, \tau)}{\partial x}=0$

(10) is the thermal symmetry condition at $x=\Delta / 2$.

The mathematical model (1-10) can be approximated both according to an implicit difference scheme of N.N. Yanenko [24, 26], and the implicit Douglas-Gunn difference scheme [27]. The resulting mathematical model is absolutely stable and convergent. Therefore, in the future, we will take the implicit difference scheme of fractional steps of 
N.N. Yanenko as the main one. According to this scheme the differential equation (1) is approximated by the scheme:

$\frac{t_{i, j, k}^{n+1 / 3}-t_{i, j, k}^{n}}{1 / 3 \triangle \tau}=\frac{\lambda}{c}\left[\Lambda_{x x}\left(t_{i, j, k}^{n+1 / 3}\right)+\Lambda_{y y}\left(t_{i, j, k}^{n+1 / 3}\right)+\Lambda_{z z}\left(t_{i, j, k}\right)\right]+\frac{1}{3} \Delta \tau \varphi$

$\frac{t_{i, j, k}^{n+2 / 3}-t_{i, j, k}^{n+1 / 3}}{1 / 3 \Delta \tau}=\frac{\lambda}{c}\left[\Lambda_{x x}\left(t_{i, j, k}^{n+1 / 3}\right)+\Lambda_{y y}\left(t_{i, j, k}^{n+2 / 3}\right)+\Lambda_{z z}\left(t_{i, j, k}^{n}\right)\right]+\frac{1}{3} \Delta \tau \varphi$

$\frac{t_{i, j, k}^{n+1}-t_{i, j, k}^{n+1 / 3}}{1 / 3 \Delta \tau}=\frac{\lambda}{c}\left[\Lambda_{x x}\left(t_{i, j, k}^{n+1 / 3}\right)+\Lambda_{y y}\left(t_{i, j, k}^{n+2 / 3}\right)+\Lambda_{z z}\left(t_{i, j, k}^{n+1}\right)\right]+\frac{1}{3} \Delta \tau \varphi$

The equation (11) is transformed:

$$
A_{1} t_{i+1, j, k}^{n+1 / 3}-C_{1} t_{i, j, k}^{n+1 / 3}+B_{1} t_{i-i, j, k}^{n+1 / 3}=-F_{1}-\frac{1}{3} \Delta \tau \varphi
$$

Where $\quad A_{1}=B_{1} \frac{\lambda \Delta \tau}{3 \mathrm{C} \triangle x^{2}} ; C_{1}=1+\frac{2 \lambda \Delta \tau}{3 \mathrm{C} \triangle x^{2}} ; \quad F_{1}=a_{2} t_{i, j,+1, k}^{n}-c_{2} t_{i, j, k}^{n}+b_{2} t_{i, j-1, k}^{n}+$ $a_{3} t_{i, j, k+1}^{n}+c_{3} t_{i, j, k}^{n}+b_{3} t_{i, j, k-1}^{n} ; \quad a_{2}=b_{2}=\frac{\lambda \Delta \tau}{3 \mathrm{C} \Delta y^{2}} ; \quad a_{3}=b_{3}=\frac{\lambda \Delta \tau}{3 \mathrm{C} \Delta z^{2}} ; c_{2}=\frac{2 \lambda \Delta \tau}{3 \mathrm{C} \Delta y^{2}}-1 ; c_{3}=$ $\frac{2 \lambda \Delta \tau}{3 \mathrm{C} \triangle \mathrm{z}^{2}}$

Similarly, for (12) and (13) we have:

$$
A_{2} t_{i, j+1, k}^{n+2 / 3}-C_{2} t_{i, j, k}^{n+2 / 3}+B_{2} t_{i, j-1, k}^{n+2 / 3}=-F_{2}-\frac{1}{3} \Delta \tau \varphi
$$

where $\quad A_{2}=B_{2} \frac{\lambda \Delta \tau}{3 \mathrm{C} \Delta y^{2}} ; C_{2}=1+\frac{2 \lambda \Delta \tau}{3 \mathrm{C} \Delta y^{2}} ; \quad F_{2}=a_{1} t_{i+1, j, k}^{n+1 / 3}-c_{1} t_{i, j, k}^{n+\frac{1}{3}}+b_{1} t_{i-1, j, k}^{n+\frac{1}{3}}+$ $a_{3} t_{i, j, k+1}^{n}-c_{3} t_{i, j, k}^{n}+b_{3} t_{i, j, k-1}^{n} ; a_{1}=b_{1}=\frac{\lambda \Delta \tau}{3 \mathrm{C} \Delta x^{2}} ; c_{1}=\frac{2 \lambda \Delta \tau}{3 \mathrm{C} \Delta x^{2}}-1 ;$

$A_{3} t_{i, j, k+1}^{n+1}-C_{3} t_{i, j, k}^{n+1}+B_{3} t_{i, j, k-1}^{n+1}=-F_{3}-\frac{1}{3} \Delta \tau-\varphi$

where $A_{3}=B_{3} \frac{\lambda \Delta \tau}{3 \mathrm{C} \triangle z^{2}} ; C_{3}=1+\frac{2 \lambda \Delta \tau}{3 \mathrm{C} \triangle z^{2}} ; \quad F_{3}=a_{1} t_{i+1, j, k}^{n+1 / 3}-\left(c_{1}+1\right) t_{i, j, k}^{n+1 / 3}+b_{1} t_{i-1, j, k}^{n+1 / 3}+$ $a_{2} t_{i, j+1, k}^{n+2 / 3}+c_{2} t_{i, j, k}^{n+2 / 3}+b_{2} t_{i, j-1, k}^{n+2 / 3}$;

The solution of equations (11-13) is:

$$
\left\{\begin{array}{l}
t_{i, j, k}^{n+1 / 3}=\alpha_{i, j, k} t_{i+1, j, k}^{n+1 / 3}+\beta_{i, j, k} \\
t_{i, j, k}^{n+2 / 3}=\vartheta_{i, j, k} t_{i+1, j, k}^{n+2 / 3}+\gamma_{i, j, k} \\
t_{i, j, k}^{n+1}=\varphi_{i, j, k} t_{i, j, k+1}^{n+1}+\psi_{i, j, k}
\end{array}\right.
$$

By analogy with (17) we write: 


$$
\left\{\begin{array}{l}
t_{i-1, j, k}^{n+1 / 3}=\alpha_{i-1, j, k} t_{i-1, j, k}^{n+1 / 3}+\beta_{i-1, j, k} \\
t_{i, j-1, k}^{n+2 / 3}=\vartheta_{i, j-1, k} t_{i, j-1, k}^{n+2 / 3}+\gamma_{i, j-1, k} \\
t_{i, j, k-1}^{n+1}=\varphi_{i, j, k-1} t_{i, j, k}^{n+1}+\psi_{i, j, k-1}
\end{array}\right.
$$

Solving together the corresponding equations (17) and (18), we find:

$$
\left\{\begin{array}{c}
t_{i-1, j, k}^{n+1 / 3}=\alpha_{i, j, k} \alpha_{i-1, j, k} t_{i+1, j, k}^{n+1 / 3}+\beta_{i, j, k} \alpha_{i-1, j, k}+\beta_{i-1, j, k} \\
t_{i, j-1, k}^{n+2 / 3}=\vartheta_{i, j, k} \vartheta_{i, j-1, k} t_{i, j+1, k}^{n+2 / 3}+\gamma_{i, j, k} \vartheta_{i, j-1, k}+\gamma_{i, j-1, k} \\
t_{i, j, k-1}^{n+1}=\varphi_{i, j, k} \varphi_{i, j, k-1} t_{i, j, k+1}^{n+1}+\psi_{i, j, k} \varphi_{i, j, k-1}+\varphi_{i, j, k-1}
\end{array}\right.
$$

After substitution the corresponding expressions (19) into (14), (15) and (16) we have:

$$
\left\{\begin{array}{c}
t_{i-1, j, k}^{n+\frac{1}{3}}=\frac{A_{1}+B_{1} \alpha_{i, j, k} \alpha_{i-1, j, k}}{C_{1}} t_{i+1, j, k}^{n+\frac{1}{3}}+\frac{B_{1} \beta_{i, j, k} \alpha_{i-1, j, k}+B_{1} \beta_{i-1, j, k}+F_{1}+\frac{1}{3} \Delta \tau \varphi}{C_{2}} \\
t_{i, j-1, k}^{n+\frac{2}{3}}=\frac{A_{2}+B_{2} \vartheta_{i, j, k} \vartheta_{i, j-1, k}}{C_{2}} t_{i, j+1, k}^{n+\frac{2}{3}}+\frac{B_{2} \gamma_{i, j, k} \vartheta_{i, j-1, k}+B_{2} \gamma_{i, j-1, k}+F_{2}+\frac{1}{3} \Delta \tau \varphi}{C_{2}} \\
t_{i, j, k-1}^{n+1}=\frac{A_{3}+B_{3} \varphi_{i, j, k} \varphi_{i, j, k-1}}{C_{3}} t_{i, j, k+1}^{n+1}+B_{3} \psi_{i, j, k} \psi_{i, j, k-1}+B_{3} \psi_{i, j, k-1}+F_{3}+\frac{1}{3} \Delta \tau \varphi
\end{array}\right.
$$

Comparing the corresponding equations (17) and (20), we find the passing coefficients:

$$
\left\{\begin{aligned}
\alpha_{i, j, k} & =\frac{A_{1}}{C_{1}-B_{1} \alpha_{i-1, j, k}} ; \beta_{i, j, k}=\frac{B_{1} \beta_{i-1, j, k}+F_{1}+1 / 3 \Delta \tau \varphi}{C_{1}-B_{1} \alpha_{i-1, j, k}} \\
\vartheta_{i, j, k} & =\frac{A_{2}}{C_{2}-B_{2} \vartheta_{i, j-1, k}} ; \gamma_{i, j, k}=\frac{B_{1} \gamma_{i, j-1, k}+F_{2}+1 / 3 \Delta \tau \varphi}{C_{2}-B_{2} \vartheta_{i, j-1, k}} \\
\varphi_{i, j, k} & =\frac{A_{3}}{C_{3}-B_{3} \varphi_{i, j, k-1}} ; \psi_{i, j, k}=\frac{B_{1} \psi_{i, j, k-1}+F_{3}+1 / 3 \Delta \tau \varphi}{C_{3}-B_{3} \varphi_{i, j, k-1}}
\end{aligned}\right.
$$

Thus, we obtained the basic equations for forecasting the temperature fields of the concrete structure during the process of its heat treatment. Numerical solution of equations (1-21) will ensure the implementation of standard temperature limitations, fulfillment of the requirements of the work performance project on the time needed to reach the designed concrete strength, as well as significant energy savings due to the beneficial use of the thermal inertia of concrete.

In addition to the method of forecasting temperature fields, we have developed a calculator based on the Microsoft Office EXCEL 2007 software, which allows accurate calculation the relative theoretical strength of concrete after heat treatment with high accuracy.

Currently, in the construction sector of the Republic of Sakha (Yakutia), when designing winter reinforced concrete works, a nomogram of concrete strength increase from TSN 12336-2007 [25] developed by the team of YPNIIS is widely used. But its small scale, the complexity of perception of nomograms (ten families of graphs on one scale ruler) and the intricate calculation scheme do not allow the concrete workers to ensure high reliability of its interpretation and interpretation of calculated data. Therefore, computer interpolation of nomograms using the MathCAD 2001i Professional program allowed us to obtain a regression equation with a high degree of correlation with the TSN 12-336-2007 nomograms 
[25]. The results of statistical calculations on assessment the adequacy of the regression equation are given in Table. 1 . The regression equation relating the average temperature and duration of heat treatment to the relative strength of concrete is as follows:

$R_{n}\left(t_{n}, \tau_{n}\right)=-17.983-0.003 \cdot \tau_{n}{ }^{2}-0.009 \tau_{n}{ }^{2}+1.639 \cdot t_{n}+0.928 \cdot \tau_{n}$,

where $R_{\mathrm{n}}$ is relative strength of concrete (the percentage of reaching the designed strength after heat treatment), \%;

$\mathrm{t}_{\mathrm{n}}$ is average temperature of heat treatment, ${ }^{\circ} \mathrm{C}$;

$\tau_{n}$ is duration of of heat treatment, $h$.

Table 1. Adequacy assessment of the regression equation

\begin{tabular}{|c|c|c|c|c|}
\hline Characteristics & Num. ${ }^{2}$ & SS model & $\begin{array}{c}\text { SS } \\
\text { residue }\end{array}$ & $\begin{array}{c}\text { F- } \\
\text { criterion }\end{array}$ \\
\hline Relative strength, $\left(\%\right.$ of $\left.\mathrm{R}_{28}\right)$ & 0.94 & 68466.79 & 4368.43 & 387.91 \\
\hline
\end{tabular}

The relative strength of concrete consists of the strengths gained during the heating process $\left(R_{\text {heat }}\right)$, isothermal exposure $R_{\text {i.e. }}$, cooling $\left(R_{c}\right)$ of the structure and can be described by the equation:

$$
R_{n}=R_{\text {heat }}+R_{\text {i.e. }}+R_{c}
$$

Taking into account the specific features of calculation the relative theoretical concrete strength according to the TSN 12-336-2007 nomograms [25], $R_{n}, R_{i . e .}$, and $R_{c}$ are calculated using the formulae:

$$
\left\{\begin{array}{l}
R_{\text {heat }}=-17.983+0.928 \cdot \tau_{\text {heat }}-0.003 \cdot \tau_{\text {heat }}^{2}+1.639 \cdot t_{\text {heat }}-0.009 \cdot t_{\text {heat }}^{2} \\
\quad R_{\text {i.e. }}=\left(-17.983+0.928 \cdot\left(\tau_{\text {heat }}+\tau_{\text {i.e. }}\right)-0.003 \cdot\left(\tau_{\text {heat }}+\tau_{\text {i.e. }}\right)^{2}+\right. \\
\left.\quad+1.639 \cdot t_{\text {i.e. }}-0.009 \cdot t_{\text {i.e. }}^{2}\right)-R_{\text {heat }} \\
\quad R_{c}=\left(-17.983+0.928 \cdot\left(\tau_{\text {heat }}+\tau_{\text {i.e. }}+\tau_{c}\right)-0.003 \cdot\left(\tau_{\text {heat }}+\tau_{\text {i.e. }}+\tau_{c}\right)^{2}+\right. \\
\left.\quad+1.639 \cdot t_{c}-0.009 \cdot t_{c}^{2}\right)-R_{\text {heat }}-R_{\text {i.e. }}
\end{array}\right.
$$

This method of finding the theoretical relative strength of concrete greatly intensifies the calculation process and almost eliminates possible errors resulting from the "human factor".

\section{Conclusions}

1. A mathematical model has been developed that adequately describes variation in temperature fields arising in concrete under the action of heat treatment at negative ambient temperature.

2. The influence of the boundary conditions on temperature field variation during a given period of time has been established

3. Computer interpolation of the TSN 12-336-2007 nomograms with the subsequent obtaining of the second order regression equation was performed.

A calculator has been developed for calculating the theoretical relative strength of concrete. This software assesses the value of the set of strength class in percentage terms depending on the duration and temperature of heat treatment. 


\section{References}

1. A. Mestnikov, A. Egorova, T. Kornilov, A. Kardasevskij, Construction Materials, 4 (2009)

2. G. Turantaev, Winter concreting of construction structures at non-traditional methods of heat treatment of concrete in thermoactive formwork (2006)

3. S. Nikolskiy, N. Vatin and O. Pertseva, J. Appl. Eng. Sci., 13, 11-8 (2015)

4. K. Semenov, Y. Barabansikov, Construction of unique buildings and constructions, 2 (2014)

5. M. Ashrabova, Ways to improve the efficiency of irrigated agriculture, 1 (2018)

6. S. Golovnev, Bulletin of volgograd state architectural and construction university. Series: construction and architecture, 31-2 (2013)

7. T. Rad'ko, Investments, construction, real estate as a material basis of modernization and innovative development of the economy (2018)

8. N. Lukuttsova, International journal of applied engineering research, 10 (2015)

9. A. Trinker, Concrete Technologies, 2 (2013)

10. I. Pavlova, M. Kocergina, M. Murakaev, Resource and enery efficient technologies in the construction complex of the region, 8 (2017)

11. A. Yudina, R. Oganyan, Architecture and Engineering, 2 (2017)

12. V. Molodin, Concreting monolithic building structures in winter conditions (2006)

13. M. Berry, J. Johnson, K. McDevitt, Cold Regions Science and Technology, 136 (2017)

14. Z. Bonić, G.T. Curčć, M. Trivunić, N. Davidović and N. Vatin, Procedia Engineer., 117, 424-435 (2015)

15. I.A. Korotchenko, E.N. Ivanov, S.S. Manovitsky, V.A. Borisova, K. V Semenov and Y.G. Barabanshchikov, Mag. Civ. Eng., 69, 56-63 (2017)

16. A.V. Bushmanova, N.V. Videnkov, K.V. Semenov, Y.G. Barabanshchikov, A.V. Dernakova, V.K. Korovina, Mag. Civ. Eng., 71, 51-60 (2017)

17. Z. Bofang, 20: Construction of Mass Concrete in Winter (2014)

18. J. Won, S. Lee, T. Park, K. Nam, Construction and Building Materials, 125 (2016)

19. A. Melnik, Procedia Engineer., 206 (2017)

20. T. Zavalisina, University news-in. Building, 7 (2002)

21. S. Golovnev, Winter concrete technology (2004)

22. Z. Zhou, P. Qiao, Cement and Concrete Composites, 94 (2018)

23. G. Turantaev, Industrial and Civil Engineering, 4 (2010)

24. N. Yanenko, The method of fractional steps for solving multidimensional problems of mathematical physics (1966)

25. TSN 12-336-2007 Concrete work at negative ambient temperatures in the Republic of Sakha (Yakutia)

26. A. Samarskij, Introduction to the theory of difference schemes (1971)

27. D. Anderson, Computational fluid mechanics and heat transfer, 1 (1990) 